\title{
Effects of dielectric inhomogeneity in polyelectrolyte solutions
}

Cite this: Soft Matter, 2013, 9, 5686

\author{
Issei Nakamura and Zhen-Gang Wang*
}

Received 3rd March 2013

Accepted 25th April 2013

DOI: $10.1039 / \mathrm{c} 3 \mathrm{sm} 50632 \mathrm{k}$

www.rsc.org/softmatter

We illustrate the effects of dielectric inhomogeneity on the statistical properties of polyelectrolytes in solution, by a lattice Monte Carlo simulation that combines the bond fluctuation model with a local algorithm for computing the electrostatic interactions. Our model accounts for the difference in the dielectric properties between the polymer backbone and the solvent. Taking the coil-globule transition of a single polyelectrolyte chain in solvent as an example, we show that the chain conformation and the degree of counterion condensation are substantially affected by the dielectric contrast.

Polyelectrolytes in solution form an important class of macromolecules that are essential in biology and colloidal science, and have been the subject of intensive theoretical and experimental investigations; see ref. 1 for an extensive review of the issues and relevant literature.

The standard model for polyelectrolytes in solution typically assumes some chain models for the polymer, such as the beadspring model, ${ }^{2}$ lattice model $^{3}$ or Gaussian thread model, ${ }^{4}$ in implicit solvents. The charges are taken to interact with Coulomb potential in a dielectric medium, with a uniform dielectric constant of the solvent. Some studies account for the solvent explicitly by treating the solvent molecules as LennardJones particles, ${ }^{5}$ but the electrostatic interactions are still included at the level of dielectric continuum with a uniform dielectric constant. In reality, however, the typically hydrophobic polymer backbone can have a very different dielectric response than the solvent, which will alter the electrostatic interactions between the charges in the vicinity of the polymer. While the use of a uniform dielectric constant of the solvent can be rationalized when the segmental concentration of the polymer is low, as in the case of dilute, good-solvent conditions, it can no longer be justified when the polymer concentration becomes high. The latter can correspond either to a concentrated bulk solution, or to a single polymer in or near a

Division of Chemistry and Chemical Engineering, California Institute of Technology, Pasadena, California 91125, USA. E-mail: zgw@caltech.edu collapsed or partially collapsed state. Even under dilute, goodsolvent conditions, the degree of charge condensation can be affected by the local dielectric response of the polymer backbone. $^{6}$

In this communication, we demonstrate the importance of including the dielectric inhomogeneity in modeling polyelectrolytes in solution by considering a single polyelectrolyte chain in solvent with no added salt. The decrease of the local dielectric response in the vicinity of the polymer leads to two effects: first, it alters (strengthens) the Coulomb interactions between the charged species, and second, it expels the counterions from the polymer-rich regions due to the unfavorable solvation energy. The combination of these two effects substantially influences the chain conformation and degree of charge condensation. For example, we show that upon decreasing the dielectric constant of the polymer backbone from that of the solvent, a collapsed polyelectrolyte chain can turn into the coil state.

For computational efficiency, we adopt a lattice formulation by combining the bond fluctuation model (BFM), ${ }^{7,8}$ a standard lattice Monte Carlo method for polymers, ${ }^{9}$ with a local algorithm developed by Maggs et al. ${ }^{\mathbf{1 0 - 1 2}}$ for computing the electrostatic interactions. This algorithm requires a computational effort of order $N,{ }^{\mathbf{1 0}}$ and can be directly implemented on a lattice, making it naturally compatible with lattice models of polymers. Furthermore, the dielectric difference between the polymer and the solvent can be easily incorporated. Thus, the lattice model used here serves as an efficient computational strategy for studying polymers and other soft matters with dielectric inhomogeneity.

We consider a single polyelectrolyte having $N$ monomer units in solvent under salt-free conditions. A fraction $f$ of these monomers are charged with an elementary charge, which we take to be equally spaced along the polymer backbone. For concreteness, we take the charge on the polymer backbone to be negative. The cations (counterions) are taken to be monovalent, so there are $f N$ cations. Because of the periodic boundary condition, the system corresponds to the cell model of a 
polyelectrolyte solution ${ }^{\mathbf{1 3}}$ at a concentration determined by the size of the simulation box, rather than to a single, isolated polyelectrolyte chain. We model the polymer conformation using the bond fluctuation model (BFM) of Shaffer ${ }^{8}$ on a simple cubic lattice with the nearest-neighbor distance $u .{ }^{14}$ Consecutive monomers of the chain are connected by the bond vectors, with allowed bond lengths, $1, \sqrt{2}$, and $\sqrt{3}$ in the lattice unit $u$. The excluded volume between the monomers is accounted for by the requirement of single occupancy of any lattice site, and empty sites are taken to be occupied by the solvent. In this study, we ignore the excluded volume between the cations and the solvent, so a cation can occupy the same site as a solvent. ${ }^{15}$ However, we do not allow simultaneous occupancy of the same lattice site by a monomer and a cation. To capture the dielectric inhomogeneity, we associate a lattice bond that connects two nearest-neighbor solvent molecules with a dielectric constant $\varepsilon_{\mathrm{s}}$, and a lattice bond that connects two nearest-neighbor monomers with a dielectric constant $\varepsilon_{\mathrm{p}}$ (Fig. 1). When a lattice bond connects a solvent molecule and a monomer, we simply take its dielectric constant to be that of the solvent on account of the dominance of ion solvation by the higher dielectric component. ${ }^{16}$ Obviously, as the conformation of the polymer changes during the Monte Carlo (MC) moves, the dielectric constant of the lattice bonds that connect the affected monomers and solvent molecules will be updated. The specifics of these rules can be modified, which will lead to slightly different results quantitatively. However, the qualitative effects we wish to elucidate will not be altered. Finally, to complete the description of the model, we assign nearest neighbor interaction energies $\sigma_{\mathrm{ps}}, \sigma_{\mathrm{pp}}$, and $\sigma_{\mathrm{ss}}$ (in unit of $k_{\mathrm{B}} T$ ) for the monomersolvent, monomer-monomer, and solvent-solvent interactions, respectively. To highlight the effect of the dielectric response of the polymer backbone, we use the same interaction parameters $\sigma_{\mathrm{ps}}$ and $\sigma_{\mathrm{pp}}$ for charged and neutral monomers. ${ }^{17}$ Similarly, all other simplifications in the description of our lattice model are adopted in order to illustrate the most important features arising from the dielectric inhomogeneity with a minimal model.

We now give a brief description of the local algorithm developed by Maggs and Rossetto. ${ }^{\mathbf{1 0}}$ Interested readers are encouraged to consult this original reference and additional references for further development and improvement of the method. ${ }^{11,12,18}$ Because our model is the first application to

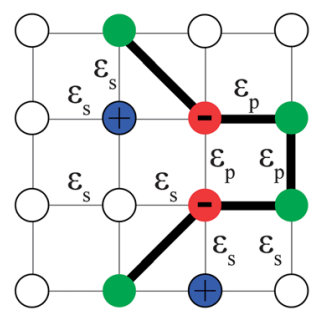

Fig. 1 A two-dimensional schematic of the bond fluctuation model illustrating the rules of assigning the dielectric constants of the lattice bonds. The green, red and blue dots represent respectively a neutral monomer, a negatively charged monomer and a cationic counterion. The solvent is represented by the blank circle. polymers, we reproduce some of the key steps in the derivation. We start with the electrostatic energy functional in the form of

$$
\mathscr{U}_{\mathrm{el}}[\boldsymbol{D}]=\int \mathrm{d} \boldsymbol{r} \frac{[\boldsymbol{D}(\boldsymbol{r})]^{2}}{2 \varepsilon_{0} \varepsilon_{\mathrm{r}}(\boldsymbol{r})},
$$

where the electric displacement field $\boldsymbol{D}(\boldsymbol{r})$ is given by the solution to the Poisson equation, $\operatorname{div} \boldsymbol{D}(\boldsymbol{r})=\rho(\boldsymbol{r}) . \rho(\boldsymbol{r})$ is the charge density, $\varepsilon_{0}$ is the vacuum permittivity, and $\varepsilon_{\mathrm{r}}(\boldsymbol{r})$ is the local dielectric constant. As we will later discretize space into lattice grids, it is convenient to scale the spatial variable by the lattice constant $u$. Further scaling the energy by $k_{\mathrm{B}} T$, the electric displacement by $e^{2} / u^{2}$, and introducing the vacuum Bjerrum length $l_{0}=e^{2} /\left(4 \pi \varepsilon_{0} k_{\mathrm{B}} T\right)$, bring eqn (1) into the dimensionless form:

$$
\mathscr{U}_{\mathrm{el}}[\boldsymbol{D}]=\frac{\alpha}{2} \int \mathrm{d} \boldsymbol{r} \frac{[\boldsymbol{D}(\boldsymbol{r})]^{2}}{2 \varepsilon_{\mathrm{r}}(\boldsymbol{r})},
$$

where $\alpha=4 \pi l_{0} / u$ and all the quantities in the integral are dimensionless; however, for conciseness, we use the same notation for these dimensionless variables as their dimensional counterparts.

The thermodynamic average of any property $S$ is given by

$$
\langle S\rangle=\frac{\int \prod_{i} \mathrm{~d} \boldsymbol{R}_{\mathrm{i}} \operatorname{Sexp}\left\{-\mathscr{U}_{0}-\mathscr{U}_{\mathrm{el}}[\boldsymbol{D}]\right\}}{\int \prod_{i} \mathrm{~d} \boldsymbol{R}_{\mathrm{i}} \exp \left\{-\mathscr{U}_{0}-\mathscr{U}_{\mathrm{el}}[\boldsymbol{D}]\right\}},
$$

where $\boldsymbol{R}_{\mathrm{i}}$ is a symbolic notation for the position vector of the $N$ monomers and $f N$ cations (counterions) and $\mathscr{U}_{0}$ represents the non-electrostatic part of the interaction. The key idea in the work of Maggs and Rossetto is to recognize that the Boltzmann factor for the electrostatic energy $\mathscr{U}_{\text {el }}[D]$ can be written as a functional integral over an auxiliary field variable $\boldsymbol{A}(\boldsymbol{r})$ by the identity, ${ }^{\mathbf{1 0}}$

$$
\begin{aligned}
\exp \left(-\mathscr{U}_{\mathrm{el}}[\boldsymbol{D}]\right)= & Z_{\mathrm{fluc}}^{-1}\left(\left\{\boldsymbol{r}_{\mathrm{i}}\right\}\right) \int \mathscr{D} \boldsymbol{A}\left\{\prod_{\boldsymbol{r}} \delta[\operatorname{div} \boldsymbol{A}(\boldsymbol{r})\right. \\
& -\rho(\boldsymbol{r})]\} \exp \left(-\mathscr{U}_{\mathrm{el}}[\boldsymbol{A}]\right) .
\end{aligned}
$$

$Z_{\text {fluc }}$ in the prefactor is given by the functional integration over the transverse vector $\boldsymbol{A}_{\mathrm{t}}(\boldsymbol{r}), \int \mathscr{D} \boldsymbol{A}_{\mathrm{t}}\left\{\prod_{\boldsymbol{r}} \delta\left[\operatorname{div} \boldsymbol{A}_{\mathrm{t}}\right]\right\} \exp \left(-\mathscr{U}_{\mathrm{el}}\left[\boldsymbol{A}_{\mathrm{t}}\right]\right)$, where $\boldsymbol{A}_{\mathrm{t}}(\boldsymbol{r})=\boldsymbol{A}(\boldsymbol{r})-\boldsymbol{D}(\boldsymbol{r})$. $Z_{\text {fluc }}$ for homogeneous dielectric media is constant, and is therefore thermodynamically inconsequential. ${ }^{10}$ For inhomogeneous dielectric media, the fluctuation potential in $Z_{\text {fluc }}$ generates the Keesom potential for the dipoledipole interaction which varies as $1 / r^{6} \cdot{ }^{11}$ In our model, this term is absorbed into the coarse-grained parameters for the molecular interactions, $\sigma_{\mathrm{ps}}, \sigma_{\mathrm{pp}}$, and $\sigma_{\mathrm{ss}}$. Using the identity eqn (4), we can evaluate the statistical average of $S$ as

$$
\begin{aligned}
\langle S\rangle= & \int \prod_{i} \mathrm{~d} \boldsymbol{R}_{\mathrm{i}} \mathscr{D A}\left\{\prod_{\boldsymbol{r}} \delta[\operatorname{div} \boldsymbol{A}(\boldsymbol{r})-\rho(\boldsymbol{r})]\right\} S \\
& \times \exp \left\{-\mathscr{U}_{0}-\mathscr{U}_{\mathrm{el}}[\boldsymbol{A}]\right\} / \int \prod_{i} \mathrm{~d} \boldsymbol{R}_{\mathrm{i}} \mathscr{D} \boldsymbol{A} \\
& \times\left\{\prod_{\boldsymbol{r}} \delta[\operatorname{div} \boldsymbol{A}(\boldsymbol{r})-\rho(\boldsymbol{r})]\right\} \exp \left\{-\mathscr{U}_{0}-\mathscr{U}_{\mathrm{el}}[\boldsymbol{A}]\right\} .
\end{aligned}
$$

Through these transformations, the average over the particle degrees of freedom becomes a double average over both the 
particle position vector and the field variable $\boldsymbol{A}(\boldsymbol{r})$. This suggests a Monte Carlo scheme that samples both degrees of freedom with the Boltzmann weight, subject to the delta function constraint (the Poisson equation). The sampling over $\boldsymbol{A}(\boldsymbol{r})$ is facilitated by noting that if $\boldsymbol{A}(\boldsymbol{r})$ is a solution to the Poisson equation, then the vector $\boldsymbol{A}^{\prime}(\boldsymbol{r})=\boldsymbol{A}(\boldsymbol{r})+\boldsymbol{C}(\boldsymbol{r})$ is also a solution for any $\boldsymbol{C}(\boldsymbol{r})$ that satisfies $\operatorname{div} \boldsymbol{C}(\boldsymbol{r})=0$; an obvious choice is $\boldsymbol{C}(\boldsymbol{r})=\operatorname{rot}$ $\boldsymbol{Q}(\boldsymbol{r})$, with $\boldsymbol{Q}(\boldsymbol{r})$ being an arbitrary vector. Thus, in a Monte Carlo scheme, starting with any particular solution to $\operatorname{div} \boldsymbol{A}(\boldsymbol{r})=\rho(\boldsymbol{r})$ for a given charge distribution, statistical sampling over $\boldsymbol{A}(\boldsymbol{r})$ involves choosing random vectors $\boldsymbol{C}(\boldsymbol{r})$ with the Boltzmann weight given in eqn (5). In our lattice model, this scheme is more conveniently implemented by using the discrete version of the integral form of the Poisson equation (the Gauss theorem). Applying the Gauss theorem to a cube of the lattice bond length that encloses site $\mathrm{m}$, we have $\sum_{\mathrm{n}} b_{\mathrm{mn}}=q_{\mathrm{m}}$, where $\mathrm{n}$ denotes the nearest neighbors of site $\mathrm{m}, q_{\mathrm{m}}$ is the charge, and $b_{\mathrm{mn}}$ is the current of the vector $\boldsymbol{A}$ through a surface of the cube that bisects the lattice bond connecting $\mathrm{m}$ and $\mathrm{n}$. By definition, $b_{\mathrm{nm}}=-b_{\mathrm{mn}}$. The energy is then evaluated as $\mathscr{U}_{\mathrm{el}}=(\alpha / 2) \sum_{\mathrm{n}} b_{\mathrm{mn}}{ }^{2} / \varepsilon_{\mathrm{mn}}$ where the summation is over all lattice bonds. The Monte Carlo sampling then involves initializing a set of $b_{\mathrm{mn}}$ for all the lattice bonds at a given charge distribution, followed by sequential updating of the group of $b_{\mathrm{mn}}$ on a plaquette in such a way that the Gauss theorem $\sum_{\mathrm{n}} b_{\mathrm{mn}}=q_{\mathrm{m}}$ is always satisfied at all the four vertices; see Fig. 2(a) for a schematic explanation. Acceptance or rejection of the update is determined by the usual Metropolis algorithm using the change in the electrostatic energy $\exp \left(-\mathscr{U}_{\mathrm{el}}[\boldsymbol{A}]\right)$ before and after the update.

For the particle positions involving the ions and the polymer, we perform the MC updates, also following the Metropolis algorithm, with the Boltzmann weighting factor of $\exp \left(-\mathscr{U}_{\mathrm{el}}[\boldsymbol{A}]\right) \times$ $\exp \left(-\boldsymbol{U}_{0}\right)$. The polymer moves are executed according to Shaffer's BFM. A scheme similar to the one for the plaquettes is used for updating the position of the charged species; see Fig. 2(b). To ensure that the electrostatic interactions are calculated accurately, we perform 100 plaquette updates for every particle position update. For the particle motion, we update the positions of the monomers with four times the frequency as the charged species. Note that the motion of the charged species also updates the lattice variable $b_{\mathrm{mn}}$ [see Fig. 2(b)]. The amplitude of the trial

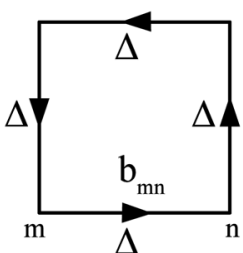

(a)

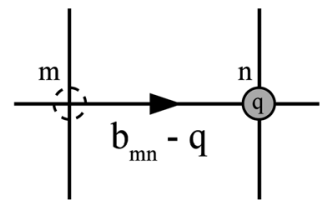

(b)
Fig. 2 Schematic illustration of the algorithm for updating (a) the lattice variable $b_{\mathrm{mn}}$ connecting the site $\mathrm{m}$ to the site $\mathrm{n}$, and $(\mathrm{b})$ the position of a charge $q$. When $b_{\mathrm{mn}}$ is increased by a random value $\Delta$, all other lattice variables on the plaquette are increased by $\Delta$ in the direction of the arrow. When a charge $q$ moves from site $\mathrm{m}$ to site $\mathrm{n}, b_{\mathrm{mn}}$ is shifted to $b_{\mathrm{mn}}-q$. Adapted from Fig. 1 of ref. 10 with permission. update $\Delta$ for the lattice variable $b_{\mathrm{mn}}$ is uniformly distributed between $-\Delta_{0}$ and $\Delta_{0}$. We have chosen $\Delta_{0}$ in the range $0.1-1.0$ so that an acceptance rate for the plaquette update between $30 \%$ and $70 \%$ is obtained, similar to that in ref. 11 . The acceptance rates are about $30 \%$ and $4 \%$ for backbone motion in good and poor solvents, respectively, and $2 \%$ for the counterions.

The size of the simulation box is $L^{3}=(40 u)^{3}$ with periodic boundary conditions. At room temperature, the vacuum Bjerrum length is $560[\AA]$, and we set the lattice unit $u$ to be $2.8[\AA]$ corresponding to the diameter of $\mathrm{K}^{+}$. This choice of lattice spacing sets the shortest contact distance between the cation and the charge on the backbone so as to obtain reasonable values for the formation energy of an ion pair. The solvent dielectric constant $\varepsilon_{\mathrm{S}}$ is 80 for water and we vary the dielectric constant of the polymer to study the effects of dielectric contrast. To characterize the chain conformation properties, we monitor the average end-to-end distance $R_{\mathrm{ee}}$ and the radius of gyration $R_{\mathrm{g}}$. As a measure for the degree of charge condensation, we calculate the average number of cations within a distance of less than or equal to $\sqrt{3} u$ of a negatively charged monomer, which we denote as $n_{\mathrm{ip}}$. The data are taken from $10^{3}$ to $10^{4}$ statistically independent parallel samples, each after an equilibration time of the order of $10^{9} \mathrm{MC}$ steps.

We first examine the behavior of a weakly charged polyelectrolyte $(N=100, f=0.1)$ in poor solvent, with an attractive monomer-monomer interaction $\sigma_{\mathrm{pp}}=-1$; the other two interaction parameters $\sigma_{\mathrm{sp}}$ and $\sigma_{\mathrm{ss}}$ are set to zero. With a uniform dielectric constant $\varepsilon_{\mathrm{p}}=\varepsilon_{\mathrm{s}}=80$, the chain is in a globular state. As we decrease the dielectric constant of the polymer, the chain conformation becomes more expanded; this is shown in Fig. 3 by the steady increase in both $R_{\text {ee }}$ and $R_{\mathrm{g}}$. To highlight the effects of the dielectric contrast, for comparison we also include results obtained by decreasing both $\varepsilon_{\mathrm{p}}$ and $\varepsilon_{\mathrm{s}}$ while keeping $\varepsilon_{\mathrm{p}}=\varepsilon_{\mathrm{s}}$ (the blue dashed line). In this case, the globular structure remains intact. However, there is a marked increase in the degree of charge condensation $n_{\mathrm{ip}}$, as shown by the blue curve in the inset of Fig. 3. The increase in ion pairing is due to increased electrostatic interaction as a result of a uniform decrease in the dielectric constant of the medium. In contrast, when decreasing $\varepsilon_{\mathrm{p}}$ at fixed $\varepsilon_{\mathrm{s}}=80$, there is an increased dielectric contrast between the polymer-rich region and the solvent. While near the polymer there is an increased attraction between the cation and the negatively charged monomer, the polymer rich region also excludes cations because of the unfavorable solvation energy of the cation in the low dielectric environment. These two competing effects, with the cation exclusion slightly outweighing the increased attraction between the unlike charges, lead to a slow decrease of $n_{\text {ip }}$ with decreasing the dielectric constant of the polymer. The exclusion of the cations from the polymer rich region results in less cations bound to the negative charge on the polymer. This, together with the increased charge repulsion between the charged monomers of the chain, results in expansion of the chain.

Next, we consider the effect of the dielectric contrast for a polyelectrolyte $(N=100$ and $f=0.5)$ in good solvents by setting $\sigma_{\mathrm{pp}}=\sigma_{\mathrm{ps}}=\sigma_{\mathrm{ss}}=0$. When $\varepsilon_{\mathrm{p}}=\varepsilon_{\mathrm{s}}=80$, the polymer is in a coil 


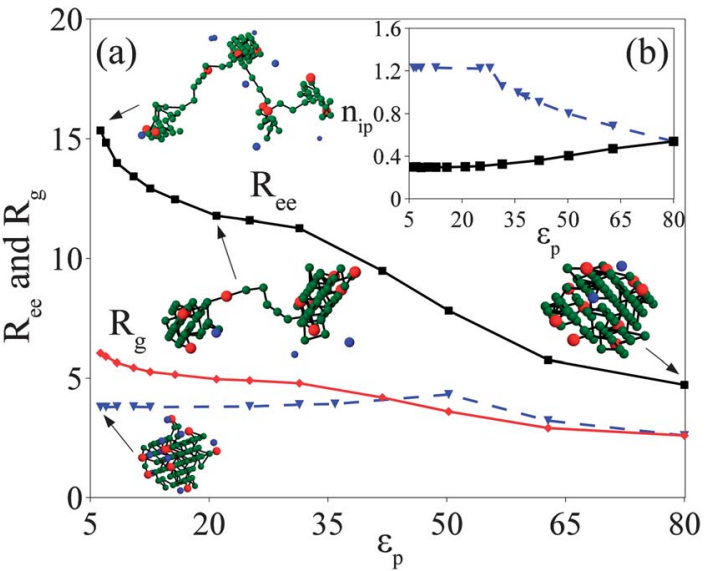

Fig. 3 (a) End-to-end distance $R_{\mathrm{ee}}$ and radius of gyration $R_{\mathrm{g}}$, and (b) degree of charge condensation $n_{\text {ip }}$ as a function of the dielectric constant of polymer $\varepsilon_{\mathrm{p}}$, for a weakly charged polyelectrolyte in a poor solvent. For comparison, we include results obtained by simultaneously changing $\varepsilon_{\mathrm{p}}$ and $\varepsilon_{\mathrm{s}}$ keeping $\varepsilon_{\mathrm{p}}=\varepsilon_{\mathrm{s}}$ (blue dashes). In the snapshots, the green, red, and blue spheres represent respectively neutral monomers, charged monomers, and counterions.

state (Fig. 4). For comparison, a charge-free neutral chain under the same solvent conditions has $R_{\mathrm{ee}} \sim 17$. As $\varepsilon_{\mathrm{p}}$ is decreased, we observe the formation of a loosely bound globule including the pearl-necklace structure with increase in counterion condensation, which leads to decrease in $R_{\mathrm{g}}$ and $R_{\mathrm{ee}}$. The increased counterion condensation is clearly a result of the increased attraction between the cations and the anionic monomers. The decreased net charge of the polymer will naturally lead to a decrease in the chain dimension. However, on closer inspection, we find that the shrinkage of the chain with decreased $\varepsilon_{\mathrm{p}}$ is largely due to the formation of cross-links among the charged sites, i.e., cations that bridge two or more neighboring charged monomers. At the lower values of $\varepsilon_{\mathrm{p}}$, the chain is a loosely bound globule, judged from the presence of relatively high density regions. Interestingly, the asphericity parameter ${ }^{\mathbf{1 9}}$ increases from 0.565 to 0.654 , as $\varepsilon_{\mathrm{p}}$ is decreased from 80 to 7 .

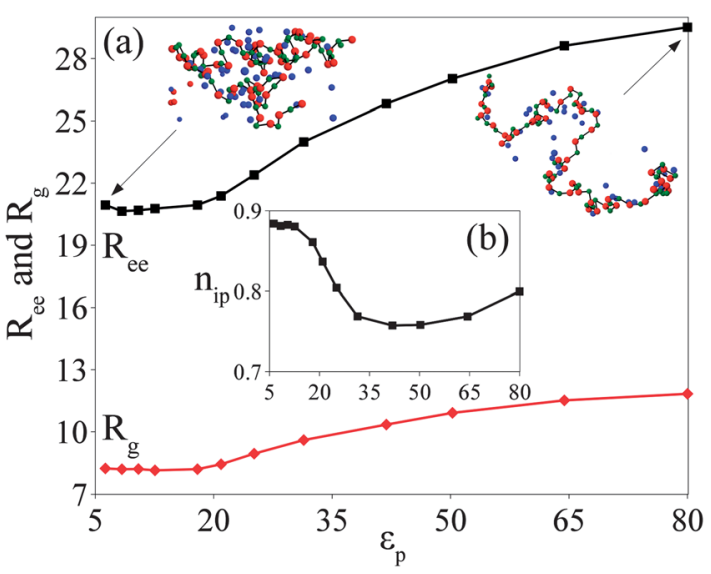

Fig. 4 (a) End-to-end distance $R_{\mathrm{ee}}$ and radius of gyration $R_{\mathrm{g}}$, and (b) degree of charge condensation $n_{\text {ip }}$ as a function of the dielectric constant of polymer $\varepsilon_{\mathrm{p}}$, for a strongly charged polyelectrolyte in a good solvent. The same color representation as in Fig. 3.
The asphericity parameter of the neutral polymer is 0.564 . Thus, the cross-links between charges formed upon decreasing the dielectric constant of the polymer makes the chain conformation more aspherical.

In summary, we have constructed a novel lattice model for a polyelectrolyte taking into account the difference in the local dielectric response between the polymer and the solvent. We find that in a poor solvent, a moderately weakly charged polyelectrolyte expands when the dielectric constant of the polymer $\varepsilon_{\mathrm{p}}$ is decreased; this is a result of the exclusion of counterions from the polymer-rich region and the increased electrostatic repulsion between the charged monomers. On the other hand, a strongly charged polyelectrolyte in good solvent becomes globular with decreased $\varepsilon_{\mathrm{p}}$ as a result of charge condensation and the formation of crosslinks among the charged species. Both are qualitative effects missed in existing simulations of polyelectrolyte solutions. The lattice model proposed here, and further improvement thereof, can serve as an efficient and powerful computational strategy for elucidating some qualitative effects in polyelectrolyte systems with spatially varying dielectric properties.

\section{Acknowledgements}

This research has been supported by the National Science Foundation, Grant no. CBET-0965812 and CHE-1040558.

\section{References}

1 A. V. Dobrynin and M. Rubinstein, Prog. Polym. Sci., 2005, 30, 1049.

2 J. L. Barrat and D. Boyer, J. Phys. II, 1993, 3, 343.

3 H. H. Hooper, H. W. Blanch and J. M. Prausnitz, Macromolecules, 1990, 23, 4820.

4 V. Ganesan and G. H. Fredrickson, Europhys. Lett., 2001, 55, 814.

5 G. Reddy and A. Yethiraj, Macromolecules, 2006, 39, 8536.

6 M. Muthukumar, J. Chem. Phys., 2004, 120, 9343.

7 I. Carmesin and K. Kremer, Macromolecules, 1988, 21, 2819.

8 J. S. Shaffer, J. Chem. Phys., 1994, 101, 4205.

9 T. Chen, M. H. Lamm and S. C. Glotzer, J. Chem. Phys., 2004, 121, 3919.

10 A. C. Maggs and V. Rossetto, Phys. Rev. Lett., 2002, 88, 196402.

11 A. C. Maggs, J. Chem. Phys., 2004, 120, 3108.

12 L. Levrel and A. C. Maggs, Phys. Rev. E: Stat., Nonlinear, Soft Matter Phys., 2005, 72, 016715.

13 A. Deshkovski, S. Obukhov and M. Rubinstein, Phys. Rev. Lett., 2001, 86, 2341.

14 We have chosen Shaffer's BFM over the original BFM consisting of connected building blocks ${ }^{7}$ for better compatibility with the local algorithm for the Coulombic interaction.

15 Because of the coarse-grain nature of the model, a solvent site is more properly interpreted as a small volume of several solvent molecules. 
16 I. Nakamura, A.-C. Shi and Z.-G. Wang, Phys. Rev. Lett., 2012, 18 A. Duncan, R. D. Sedgewick and R. D. Coalson, Phys. Rev. E: 109, 257802. Stat., Nonlinear, Soft Matter Phys., 2005, 71, 046702.

17 I. Nakamura, N. P. Balsara and Z.-G. Wang, Phys. Rev. Lett., 19 M. Bishop and W. Smith, J. Chem. Phys., 1991, 95, 2011, 107, 198301. 3804. 